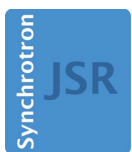

JOURNAL OF SYNCHROTRON RADIATION

ISSN 1600-5775

Received 4 July 2017

Accepted 28 September 2017

Edited by E. Plönjes, DESY, Germany

Keywords: hard X-ray free-electron laser; split-and-delay optics; multi-crystal optics; $\mathrm{X}$-ray photon correlation spectroscopy.

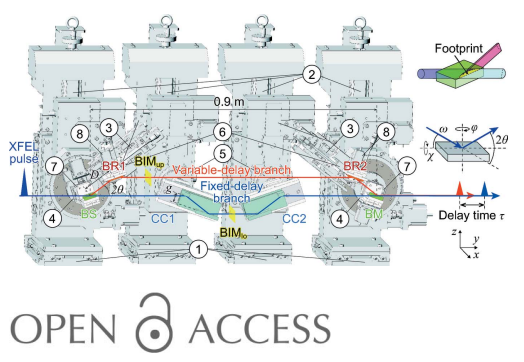

\section{Performance of a hard X-ray split-and-delay optical system with a wavefront division}

\author{
Takashi Hirano, ${ }^{\mathrm{a} *}$ Taito Osaka, ${ }^{\mathrm{a}, \mathrm{b}}$ Yuki Morioka, ${ }^{\mathrm{a}}$ Yasuhisa Sano, ${ }^{\mathrm{a}}$ \\ Yuichi Inubushi, ${ }^{\mathrm{b}, \mathrm{c}}$ Tadashi Togashi, ${ }^{\mathrm{b}, \mathrm{c}}$ Ichiro Inoue, ${ }^{\mathrm{b}}$ Satoshi Matsuyama, ${ }^{\mathrm{a}}$ \\ Kensuke Tono, ${ }^{b, c}$ Aymeric Robert, ${ }^{d}$ Jerome B. Hastings, ${ }^{e}$ Kazuto Yamauchi ${ }^{a}$ and \\ Makina Yabashi ${ }^{\text {b }}$
}

\footnotetext{
${ }^{a}$ Department of Precision Science and Technology, Graduate School of Engineering, Osaka University, 2-1 Yamada-oka, Suita, Osaka 565-0871, Japan, ${ }^{\mathbf{b}}$ RIKEN SPring-8 Center, 1-1-1 Kouto, Sayo-cho, Sayo-gun, Hyogo 679-5148, Japan, Japan Synchrotron Radiation Research Institute (JASRI), 1-1-1 Kouto, Sayo-cho, Sayo-gun, Hyogo 679-5198, Japan, dLinac Coherent Light Source, SLAC National Accelerator Laboratory, 2575 Sand Hill Road, Menlo Park, CA 94025, USA, and ${ }^{\mathbf{e}}$ SLAC National Accelerator Laboratory, 2575 Sand Hill Road, Menlo Park, CA 94025, USA.

*Correspondence e-mail: hirano@up.prec.eng.osaka-u.ac.jp
}

The performance of a hard X-ray split-and-delay optical (SDO) system with a wavefront division scheme was investigated at the hard X-ray free-electron laser facility SACLA. For the wavefront division, beam splitters made of edgepolished perfect $\mathrm{Si}(220)$ crystals were employed. We characterized the beam properties of the SDO system, and investigated its capabilities for beam manipulation and diagnostics. First, it was confirmed that shot-to-shot noninvasive diagnostics of pulse energies for both branches in the SDO system was feasible. Second, nearly ideal and identical focal profiles for both branches were obtained with a spot size of $\sim 1.5 \mu \mathrm{m}$ in full width at half-maximum. Third, a spatial overlap of the two focused beams with a sub- $\mu \mathrm{m}$ accuracy was achieved by fine tuning of the SDO system. Finally, a reliable tunability of the delay time between two pulses was confirmed. The time interval was measured with an $\mathrm{X}$-ray streak camera by changing the path length of the variable-delay branch. Errors from the fitted line were evaluated to be as small as $\pm 0.4 \mathrm{ps}$ over a time range of $60 \mathrm{ps}$.

\section{Introduction}

The advent of hard X-ray free-electron lasers (XFELs) with ultra-high intensity, excellent transverse coherence and pulse duration of less than $10 \mathrm{fs}$ has offered new opportunities in revealing ultrafast dynamics in matter at angstrom spatial and femtosecond time scales. New insights into ultrafast phenomena have been obtained via an optical pump and XFEL probe technique in which the prompt optical laser pulse excites electrons at a valence level and then the delayed XFEL pulse probes the following dynamics, such as chemical reactions (Kim et al., 2015), atomic and magnetic ordering/disordering (Dean et al., 2016) and phonons (Clark et al., 2013; Trigo et al., 2013), as a function of the delay time between the pump and probe pulses. X-ray-induced damage processes associated with the core-excitation and following multiple ionization have been studied through an XFEL pump and XFEL probe technique (Inoue et al., 2016) with an accelerator-based dual XFEL pulse generation (DXPG) method (Hara et al., 2013). In this case, a typical delay time range is from a few femtoseconds to hundreds of femtoseconds. With an extended delay range up to sub-picoseconds or nanoseconds, spontaneous fluctuations in diverse systems at 
angstrom to nanometer spatial scales will be unveiled through, for example, an X-ray photon correlation spectroscopy (XPCS; Grübel et al., 2007; Gutt et al., 2009) method using XFEL pulse pairs, although these ranges are inaccessible with the accelerator-based DXPG methods (Hara et al., 2013; Marinelli et al., 2015).

An optics-based DXPG system, which is also called a splitand-delay optical (SDO) system, is highly useful for extending the delay range. A single XFEL pulse is divided into two pulses by a beam splitter. The split pulses propagate through two delay branches in the SDO system, and finally are recombined at a sample with a time delay. The delay time is determined by the path length difference between the delay branches, resulting in a jitter-free delay time control through precise adjustments of the path length difference. In the hard $\mathrm{X}$-ray regime, several SDO layouts based on grazing-incidence multilayer mirrors (Roling et al., 2014), diffraction gratings (David et al., 2015) and perfect crystals (Roseker et al., 2009, 2011; Stetsko et al., 2013; Sakamoto et al., 2017) have been proposed, and some systems have been tested at synchrotron and/or XFEL facilities. In particular, crystal-based SDO systems can easily generate a large delay time of more than $100 \mathrm{ps}$ with a reasonably small apparatus, because of a large deflection angle (greater than several degrees) for Bragg diffractions. Furthermore, monochromatic XFEL pulses generated with the crystal-based SDO system are preferable for achieving high resolutions in typical X-ray diffraction experiments and for increasing the visibility of speckle patterns at a high scattering vector range in coherence-based experiments including XPCS. However, the crystal-based SDO systems that have been previously reported operate only at discrete photon energies, due to special optical configurations with high symmetry, such as $45^{\circ}$ diffraction, $90^{\circ}$ diffraction or multi-beam case diffraction. The development of an SDO system with wide and continuous photon energy ranges has been in high demand for broad applications such as resonance experiments of various elements.

We have developed a versatile SDO system operating in a wide and continuous photon energy range from 6.5 to $11.5 \mathrm{keV}$, and tested it at SPring8 (Osaka et al., 2016). In the test, a spectral division scheme with $10 \mu \mathrm{m}$ thick $\mathrm{Si}(220)$ crystals (Osaka et al., 2013) was employed. Although the basic performance of this system was successfully confirmed, we found that a wavefront of the split beam reflected by the thin crystals was distorted, possibly because the lattice in the thin crystals were slightly bent due to the weakness of the structure against internal and external stresses. As a result, the focal profile in this thincrystal branch was broadened. In this paper, we present the results of performance tests of the SDO system that uses a wavefront division scheme with an edgepolished thick crystal, conducted at an XFEL facility, SPring-8 Angstrom Compact free-electron LAser (SACLA; Ishikawa et al., 2012). We characterized the beam properties of the SDO system, and investigated its capabilities for beam manipulation and diagnostics.

\section{Conceptual design of the SDO system}

Fig. 1(a) shows the schematics of the SDO system consisting of six separate $\mathrm{Si}(220)$ crystals in the vertical scattering geometry; a beam splitter (BS), two beam reflectors (BRs), a beam merger (BM) in the variable-delay (upper) branch, and a pair of speckle-free channel-cut crystals (CCs; Hirano et al., 2016) in the fixed-delay (lower) branch. In the wavefront division scheme, an X-ray wavefront is laterally divided into reflected (upper) and unreflected (lower) beams at the edge of the BS, as illustrated in Fig. 1(b). The spectrum of each split beam can be independently selected, allowing for the production of double pulses with the same photon energies. Intensities of both branches could also be balanced because the split beams experience the same numbers of reflections with each other. Additionally, it is easy to control the intensity ratio by translating the edge positions of the $\mathrm{BS}$ and $\mathrm{BM}$.

The mechanics of the SDO system [Fig. 1(a) and Table 1] followed those described by Osaka et al. (2016). For controlling the crystals in the upper branch, motorized precision stages for $\omega$ and $\chi$ axes [see Fig. 1(c)] are utilized. A wide range of photon energies are covered continuously by changing the incident angle $\omega$ of the crystals. The photon energy range is $6.5-11.5 \mathrm{keV}$ for the lower branch and above $3.2 \mathrm{keV}$ for the upper branch.

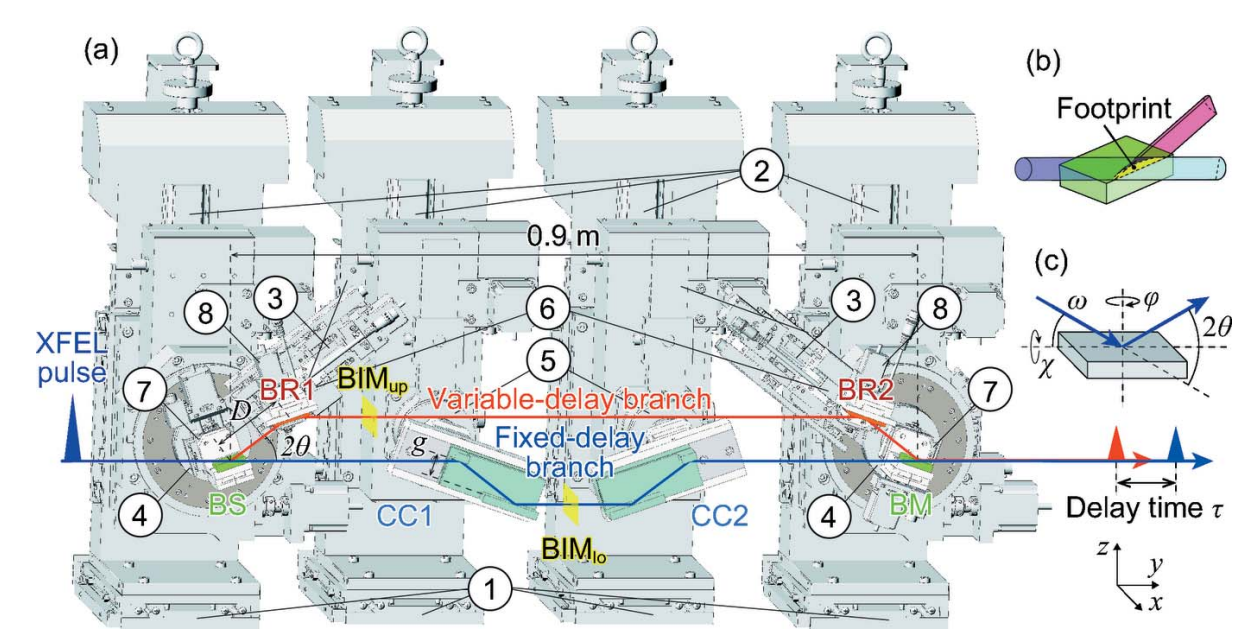

Figure 1

(a) Illustration of the mechanics of the SDO system. Crystals BS, BRs, BM and CCs represent the beam splitter, beam reflectors, beam merger and channel-cut crystals, respectively. Translation stages in the (1) $x$, (2) $z$ and (3) $D$ directions. Rotation stages around the (4) $\omega-2 \theta$ axis for BS/BM, (5) $\omega$ axis for CCs and (6) $\omega$ axis for BRs. Swivel stages around the $\chi$ axis for (7) BS/BM and (8) BRs. The range of $D$ is set to $57-127 \mathrm{~mm}$. Beam intensity monitors $\left(\mathrm{BIM}_{\mathrm{up}}\right.$ and $\left.\mathrm{BIM}_{\mathrm{lo}}\right)$ are inserted into the two delay branches. Conceptual sketches of $(b)$ wavefront division and $(c)$ four general axes in X-ray diffraction. 
Table 1

Stage resolutions in half-step feed mode.

\begin{tabular}{llll}
\hline $\begin{array}{l}\text { Number in } \\
\text { Fig. } 1(a)\end{array}$ & Crystal & Axis & Resolution \\
\hline 1 & BS, BM, CCs & $x$ & $1.0 \mu \mathrm{m}$ \\
2 & BS, BM, CCs & $z$ & $1.0 \mu \mathrm{m}$ \\
3 & BRs & $D$ & $0.25 \mu \mathrm{m}$ \\
4 & BS, BM & $\omega$ & $24.2 \mathrm{nrad}$ \\
& BRs & $2 \theta$ & $34.9 \mu \mathrm{rad}$ \\
5 & CCs & $\omega$ & $24.2 \mathrm{nrad}$ \\
6 & BRs & $\omega$ & $0.70 \mu \mathrm{rad}$ \\
7 & BS, BM & $\chi$ & $8.38 \mu \mathrm{rad} \dagger$ \\
8 & BRs & $\chi$ & $23.4 \mu \mathrm{rad}$ \\
\hline
\end{tabular}

$\dagger$ The resolution in the $\chi$ axis of BS/BM was enhanced to $83.8 \mathrm{nrad}$ by the operation in $1 / 200$-step feed mode.

The path length of the upper branch is controlled by translating the BRs along the beam axes with linear stages connected to the $2 \theta$ arms. The delay time $\tau$ is written as

$$
\begin{aligned}
\tau & =\frac{2\left(1-\cos 2 \theta_{\mathrm{B}}^{\text {up }}\right)}{c} D-\frac{2 g\left(1-\cos 2 \theta_{\mathrm{B}}^{\text {lo }}\right)}{c \sin \theta_{\mathrm{B}}^{\text {lo }}} \\
& =A\left(\theta_{\mathrm{B}}^{\text {up }}\right) D-B\left(\theta_{\mathrm{B}}^{\text {lo }}\right),
\end{aligned}
$$

where $D$ is the path length between adjacent BS/BM and BRs, $\theta_{\mathrm{B}}^{\text {up }}\left(\theta_{\mathrm{B}}^{\text {lo }}\right)$ is the Bragg angle for the upper (lower) branch, $g=$ $30 \mathrm{~mm}$ is the channel width of the CCs, and $c$ is the speed of light. The coefficient $A(B)$ is a function of the Bragg angle, i.e. the photon energy, for the upper (lower) branch. The translation range of the BRs was $57<D<127 \mathrm{~mm}$. For example, the corresponding range of the delay time is $-50<$ $\tau<+47$ ps at $10 \mathrm{keV}\left(\theta_{\mathrm{B}}^{\text {up }}=\theta_{\mathrm{B}}^{\text {lo }}=18.8^{\circ}\right)$, while the maximum delay can be extended up to $220 \mathrm{ps}$ at $6.5 \mathrm{keV}\left(\theta_{\mathrm{B}}^{\text {up }}=\theta_{\mathrm{B}}^{\text {lo }}=\right.$ $\left.29.8^{\circ}\right)$ in this system. The delay time is controlled with a resolution of $<1 \mathrm{fs}$ over the photon energy range of 6.5$11.5 \mathrm{keV}$.

\section{Performance characterization}

We tested the SDO system at the hard X-ray beamline BL3 of SACLA (Tono et al., 2013) to study the following targets: (i) Shot-to-shot non-invasive measurement of the pulse energies for both branches simultaneously. This capability is required for accurate analysis of experimental data to compensate possible fluctuation of XFEL pulse energies. (ii) Characterization of the focused beam profile of each branch. (iii) Spatial control of the relative beam positions on the focal plane. We note that precise angular tuning finer than $0.1 \mu \mathrm{rad}$ is required for assuring an overlap between the focused beams in the present setup, as described in \$3.2. (iv) Temporal control of the interval between two pulses, which is one of the most important targets of the SDO system, and can be characterized only with femtosecond XFEL pulses.

The experiment was performed with $10 \mathrm{keV}$ monochromatic XFEL pulses through a $\mathrm{Si}(111)$ double-crystal monochromator (DCM) at a repetition rate of $30 \mathrm{~Hz}$. The SDO system was installed $\sim 100 \mathrm{~m}$ downstream from the last undulator segment. The edge positions of the BS and BM were adjusted along the horizontal direction so as to transport the half portion of the incident beam profile into the upper branch. The exit beams from the SDO system were focused with a $1 \mu \mathrm{m}$ focusing mirror system (Yumoto et al., 2012) located $14 \mathrm{~m}$ downstream. Three inline diagnostic modules were implemented to measure the pulse energy of the incident beam (BIM0; Tono et al., 2011) and those of the two delay branches $\left(\mathrm{BIM}_{\mathrm{up}}\right.$ and $\left.\mathrm{BIM}_{\mathrm{lo}}\right)$ in the SDO system, as shown in Fig. 1(a). The modules $\mathrm{BIM}_{\mathrm{up}}$ and $\mathrm{BIM}_{\mathrm{lo}}$ consisted of a thin polyimide film scatter with a thickness of $50 \mu \mathrm{m}$, two photodiodes, and lead shields with $\sim 8 \mathrm{~mm}$ holes for XFEL transmission. They were also used as beam stoppers for a single branch operation mode by shifting their lateral positions. Pulse energies of the exit beams were measured with an optional PIN photodiode $\left(\mathrm{PD}_{\text {mirror }}\right)$ placed in front of the focusing system. The delay time was measured with an X-ray streak camera (HAMAMATSU, C4575-03) placed $17 \mathrm{~m}$ downstream from the SDO system.

We coarsely adjusted the position and the angle of the upper beam by monitoring the beam positions at the detector planes of two beam profile monitors BPM1 (HAMAMATSU, ORCA flash with a $5.0 \times$ magnification lens system, $1.25 \mu \mathrm{m}$ pixel $\left.^{-1}\right)$ and BPM2 (4.0 $\mu \mathrm{m}$ pixel $^{-1}$; Kameshima et al., 2016), located $0.4 \mathrm{~m}$ and $16 \mathrm{~m}$ downstream of the SDO system, respectively. The incident angles $\omega$ of all crystals were precisely aligned to give the same photon energies for the upper and lower beams with an accuracy of $<50 \mathrm{meV}$. Figs. 2(a) and $2(b)$ display averaged beam profiles measured by BPM1 and BPM2, respectively. We did not observe unwanted speckles from the crystals in the SDO system, whereas weak
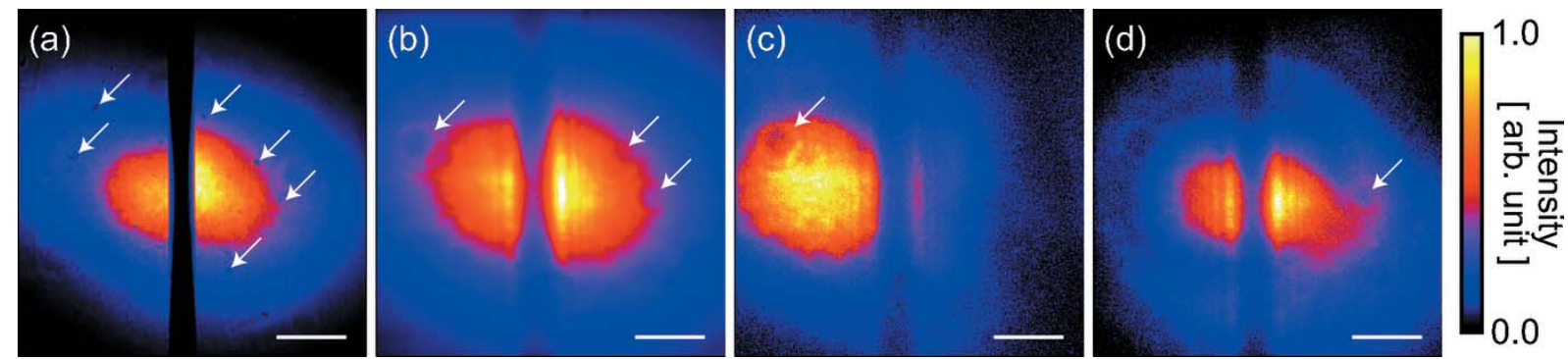

Figure 2

Averaged profiles of the exit beams measured with $(a)$ BPM1 and $(b)$ BPM2. The left (right) part of the profile in each panel shows the exit beam from the upper (lower) branch. Speckles as shown by the white arrows were caused by a Be window used for the upstream beamline optics and dust on the polyimide film of BIMs. $(c, d)$ Typical single-shot beam profiles measured with BPM2. Differences between the profiles are due to the shot-to-shot profile and positional variations of the incident XFEL beams. The scale bars represent a length of $0.2 \mathrm{~mm}$. 

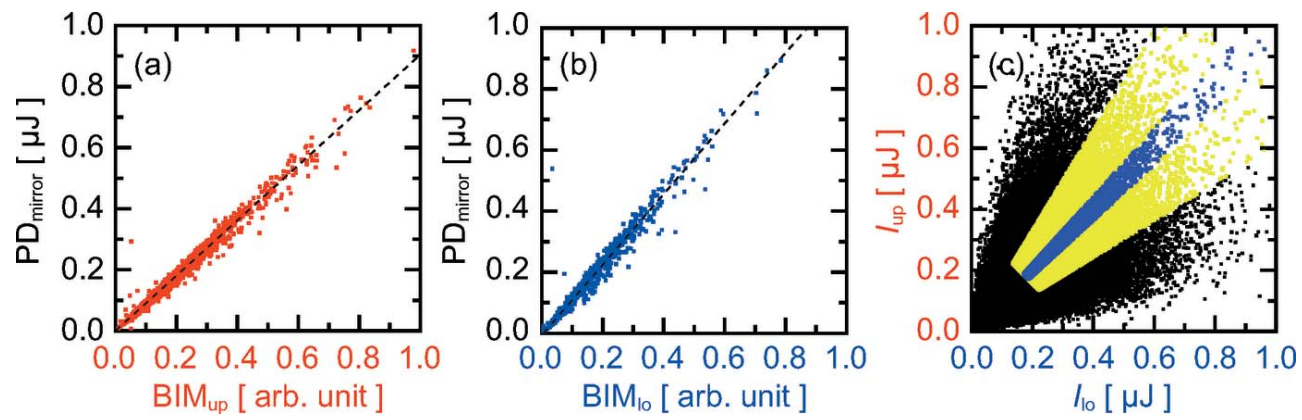

Figure 3

Shot-to-shot pulse energies of $(a)$ upper and $(b)$ lower branches for $\sim 1300$ shots. The intensities measured with $\mathrm{PD}_{\text {mirror }}$ are plotted as a function of that with $\mathrm{BIM}_{\mathrm{up}}$ and $\mathrm{BIM}_{\mathrm{lo}}$ in $(a)$ and $(b)$, respectively. (c) Correlation between shot-to-shot pulse energies $I_{\text {up }}$ and $I_{\mathrm{lo}}$ for 100000 shots. Yellow points (26\% of the total events) satisfy the condition of the normalized difference $\left|R_{\mathrm{d}}\right|=\left|2\left(I_{\mathrm{up}}-I_{\mathrm{lo}}\right) /\left(I_{\mathrm{up}}+I_{\mathrm{lo}}\right)\right|<0.5$ and $I_{\mathrm{up}}+I_{\mathrm{lo}}>0.35 \mu \mathrm{J}$, while purple points $(5.8 \%$ of the total events) show $\left|R_{\mathrm{d}}\right|<0.1$ and $I_{\mathrm{up}}+I_{\mathrm{lo}}>0.35 \mu \mathrm{J}$.

diffraction fringes from the edge of the BS and/or BM were obtained.

\subsection{Shot-to-shot pulse energy diagnostics}

We investigated the feasibility of shot-to-shot non-invasive measurement of the pulse energies for the upper and lower branches simultaneously. For this purpose, we compared pulse energies measured with $\mathrm{BIM}_{\mathrm{up}}\left(\mathrm{BIM}_{\mathrm{lo}}\right)$ in the SDO system with those given by $\mathrm{PD}_{\text {mirror }}$ in the single branch operation mode, where the beam path of the lower (upper) branch was blocked. Excellent linearities were obtained for both cases, as plotted in Figs. 3(a) and 3(b). Errors from the fitted line were $6 \%$ in the relative standard deviation. The values measured with $\mathrm{BIM}_{\text {up }}\left(\mathrm{BIM}_{\mathrm{lo}}\right)$ were converted to the pulse energies $I_{\text {up }}\left(I_{\mathrm{lo}}\right)$ based on the fitting equation displayed as a dashed line in Fig. 3(a) (Fig. 3b). Fig. 3(c) shows a correlation between $I_{\text {up }}$ and $I_{\mathrm{lo}}$ for 100000 events. The ratio of pulse energies between the upper and lower branches was widely distributed shot-by-shot, whereas it was almost unity on average. The yellow points in Fig. 3(c) (26\% of the total events) satisfy the condition of the normalized difference $\left|R_{\mathrm{d}}\right|=\mid 2\left(I_{\mathrm{up}}-I_{\mathrm{lo}}\right) /$ $\left(I_{\mathrm{up}}+I_{\mathrm{lo}}\right) \mid<0.5$ and $I_{\mathrm{up}}+I_{\mathrm{lo}}>0.35 \mu \mathrm{J}$, while the purple points (5.8\% of the total events) show $\left|R_{\mathrm{d}}\right|<0.1$ and $I_{\text {up }}+I_{\mathrm{lo}}>$ $0.35 \mu \mathrm{J}$. The distribution originated from large shot-to-shot variations in profile and position of the incident $\mathrm{Si}(111)$ monochromatic beams as shown in Figs. 2(c) and 2(d). Note that the spectral fluctuations of the incident XFEL beam could not vary the ratio of the pulse energies, because both branches used the same photon energies in the wavefront division scheme.

The averaged pulse energies of the incidence beam and the sum of exit beams from both branches were $7.9 \mu \mathrm{J}$ and $0.38 \mu \mathrm{J}$, respectively, indicating a throughput of $4.8 \%$. Note that the throughput reflected not only an efficiency of the SDO system but also the transmissivities of the existing air pass of $\sim 2 \mathrm{~m}$ $(\sim 30 \%)$ and the beamline components with additional polyimide windows on vacuum ducts $(\sim 70 \%)$. Considering the total transmissivity of $20 \%$ measured with removing the SDO system, we evaluated the efficiency of the SDO system to be $24 \%$, which was slightly smaller than the calculated value of $36 \%$. The decrease in the measured efficiency may originate from slight angular errors on the optical elements and imperfection of the crystal lattice near the edges of the BS and $\mathrm{BM}$.

\subsection{Focusing characteristics}

An averaged focal profile of each branch was measured by the knife-edge scanning method, as shown in Fig. 4. The focal spot sizes of the upper and lower beams were $1.29 \mu \mathrm{m}(\mathrm{H}) \times$ $1.81 \mu \mathrm{m}(\mathrm{V})$ and $1.49 \mu \mathrm{m}(\mathrm{H}) \times 1.42 \mu \mathrm{m}(\mathrm{V})$ in full width at half-maximum, respectively, which almost agreed with those given in the ideal condition. This result showed that the wavefront distortion previously observed for the thin-crystal branch in the spectral division scheme was sufficiently reduced in the present case. Furthermore, Fig. 4 shows an excellent overlap of the two branches with an accuracy of $<0.2 \mu \mathrm{m}$. This was accomplished by precise tuning of the angles of the
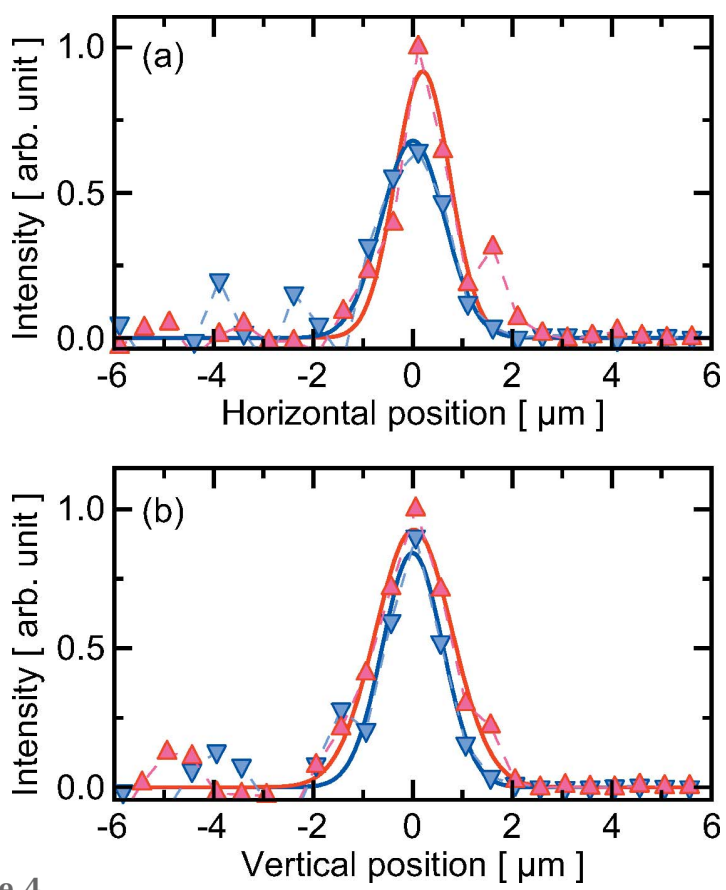

Figure 4

Averaged profiles of the focused beams in the $(a)$ horizontal and $(b)$ vertical directions. Red triangles and blue inverse triangles represent upper and lower branches, respectively. Each data point was measured using an average of ten shots. Solid curves indicate the fitted lines with the Gaussian functions. 
crystals finer than $75 \mathrm{nrad}$ and $0.23 \mu \mathrm{rad}$ for the $\omega$ and $\chi$ axes, respectively, which was imposed by a typical source size of 60-80 $\mu \mathrm{m}$ and the distance between the source and the SDO system (Osaka et al., 2016). Note that the focal positions and profiles vary shot-by-shot, which can be characterized by using a state-of-the-art high-resolution X-ray camera.

\subsection{Delay time control}

We measured the delay time using the X-ray streak camera (Fig. 5a). The temporal window, pixel resolution and effective time resolution under these experimental conditions were $200 \mathrm{ps}, 0.47 \mathrm{ps}$ and $\sim 3 \mathrm{ps}$, respectively. Averaged delay times between two pulses are plotted as a function of the setting path length $D$ in Fig. 5(b). We obtained good linearity with a fitting error of $\pm 0.5 \%$. Errors from the fitted line were less than 0.4 ps over the time range of $\sim 60$ ps. The slope values of the fitted and calculated lines based on equation (1) agreed with a small deviation of $1.0 \%$. This error was explained by $0.07^{\circ}$ angular deviations of both $2 \theta$ arms from the ideal positions. The offset of delay between measured and calculated
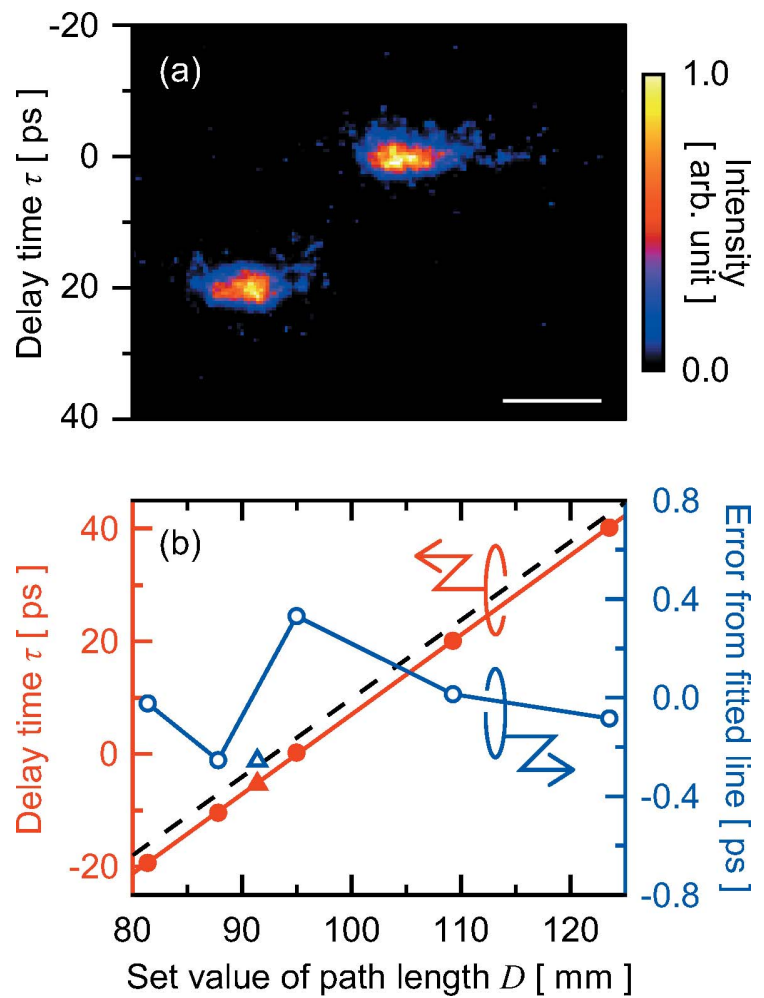

Figure 5

(a) Typical split-and-delayed pulses measured with the X-ray streak camera. The delay time between two pulses was set to be 20 ps. Profiles in the horizontal and vertical directions correspond to spatial (horizontal) and temporal information, respectively. The width of the temporal distribution of each pulse, $\sim 3 \mathrm{ps}$, was due to the effective resolution of the camera. The scale bar represents a length of $0.4 \mathrm{~mm}$. (b) Delay time as a function of the setting path length between adjacent BS/BM and BRs. Averaged delay times at each set value of the path length are displayed as closed red circles. The solid red line represents the fitted line. Open blue circles indicate the errors from the fitted line. The dashed black line represents the calculated line in the ideal condition. Closed red and open blue triangles indicate averaged delay time and the error from the fitted line, respectively, measured after the delay scan plotted as circles. values was $\sim 3 \mathrm{ps}$, which corresponds to the deviations in the setting path length $D$ and in the channel width $g$.

\section{Conclusion}

The performance tests of the SDO system with wavefront division scheme based on $\mathrm{Si}(220)$ diffraction of the perfect crystals, operating at a photon energy of $6.5-11.5 \mathrm{keV}$, were conducted with $10 \mathrm{keV}$ XFELs at SACLA. We confirmed that shot-to-shot non-invasive pulse energy diagnostics for the two delay branches was feasible while the ratio of the pulse energies fluctuated strongly. We successfully obtained nearly ideal and identical focal beams with a spot size of $\sim 1.5 \mu \mathrm{m}$, with an excellent spatial superimposition in a sub-micrometer accuracy. The slope of the fitted line to the measured delay time as a function of the setting path length $D$ was in good agreement with the calculated line.

We are planning to upgrade the SDO system with dedicated mechanics to enable more robust operation with reduction in the angular changes caused by the delay scan, while extending the range of the photon energy and the delay time. Also, the employment of an amplitude division scheme could generate double XFEL pulses with more identical properties without fluctuation of the ratio of the pulse energies, which requires ultrathin perfect $\mathrm{Si}(220)$ crystals with a thickness of $2 \mu \mathrm{m}$ as BS and BM.

\section{Acknowledgements}

The authors would like to thank the staff of SPring-8 and SACLA facilities for their continuous support. We also wish to thank Drs Diling Zhu, Matthieu Chollet and Takahiro Sato at the LCLS in the USA, and Dr Wojciech Roseker at DESY in Germany, for fruitful discussions. Characterization of the crystal optics in the SDO system was carried out at BL29XUL of SPring-8 with the approval of RIKEN (Proposal Nos. 20140013 and 20150075).

\section{Funding information}

The following funding is acknowledged: Special Postdoctoral Researcher Program of RIKEN; JSPS KAKENHI (award No. JP17J01667); Program for Leading Graduate Schools: 'Interactive Materials Science Cadet Program'.

\section{References}

Clark, J. N., Beitra, L., Xiong, G., Higginbotham, A., Fritz, D. M., Lemke, H. T., Zhu, D., Chollet, M., Williams, G. J., Messerschmidt, M., Abbey, B., Harder, R. J., Korsunsky, A. M., Wark, J. S. \& Robinson, I. K. (2013). Science, 341, 56-59.

David, C., Karvinen, P., Sikorski, M., Song, S., Vartiainen, I., Milne, C. J., Mozzanica, A., Kayser, Y., Diaz, A., Mohacsi, I., Carini, G. A., Herrmann, S., Färm, E., Ritala, M., Fritz, D. M. \& Robert, A. (2015). Sci. Rep. 5, 7644.

Dean, M. P. M., Cao, Y., Liu, X., Wall, S., Zhu, D., Mankowsky, R., Thampy, V., Chen, X. M., Vale, J. G., Casa, D., Kim, J., Said, A. H., Juhas, P., Alonso-Mori, R., Glownia, J. M., Robert, A., Robinson, J., Sikorski, M., Song, S., Kozina, M., Lemke, H., Patthey, L., Owada, S., Katayama, T., Yabashi, M., Tanaka, Y., Togashi, T., Liu, J., Rayan 
Serrao, C., Kim, B. J., Huber, L., Chang, C.-L., McMorrow, D. F., Först, M. \& Hill, J. P. (2016). Nat. Mater. 15, 601-605.

Grübel, G., Stephenson, G. B., Gutt, C., Sinn, H. \& Tschentscher, Th. (2007). Nucl. Instrum. Methods Phys. Res. B, 262, 357-367.

Gutt, C., Stadler, L.-M., Duri, A., Autenrieth, T., Leupold, O., Chushkin, Y. \& Grübel, G. (2009). Opt. Express, 17, 55-61.

Hara, T., Inubushi, Y., Katayama, T., Sato, T., Tanaka, H., Tanaka, T., Togashi, T., Togawa, K., Tono, K., Yabashi, M. \& Ishikawa, T. (2013). Nat. Commun. 4, 2919.

Hirano, T., Osaka, T., Sano, Y., Inubushi, Y., Matsuyama, S., Tono, K., Ishikawa, T., Yabashi, M. \& Yamauchi, K. (2016). Rev. Sci. Instrum. 87, 063118.

Inoue, I., Inubushi, Y., Sato, T., Tono, K., Katayama, T., Kameshima, T., Ogawa, K., Togashi, T., Owada, S., Amemiya, Y., Tanaka, T., Hara, T. \& Yabashi, M. (2016). Proc. Natl Acad. Sci. USA, 113, 1492-1497.

Ishikawa, T., Aoyagi, H., Asaka, T., Asano, Y., Azumi, N., Bizen, T., Ego, H., Fukami, K., Fukui, T., Furukawa, Y., Goto, S., Hanaki, H., Hara, T., Hasegawa, T., Hatsui, T., Higashiya, A., Hirono, T., Hosoda, N., Ishii, M., Inagaki, T., Inubushi, Y., Itoga, T., Joti, Y., Kago, M., Kameshima, T., Kimura, H., Kirihara, Y., Kiyomichi, A., Kobayashi, T., Kondo, C., Kudo, T., Maesaka, H., Maréchal, X. M., Masuda, T., Matsubara, S., Matsumoto, T., Matsushita, T., Matsui, S., Nagasono, M., Nariyama, N., Ohashi, H., Ohata, T., Ohshima, T., Ono, S., Otake, Y., Saji, C., Sakurai, T., Sato, T., Sawada, K., Seike, T., Shirasawa, K., Sugimoto, T., Suzuki, S., Takahashi, S., Takebe, H., Takeshita, K., Tamasaku, K., Tanaka, H., Tanaka, H., Tanaka, R., Togashi, T., Togawa, K., Tokuhisa, A., Tomizawa, H., Tono, K., Wu, S., Yabashi, M., Yamaga, M., Yamashita, A., Yanagida, K., Zhang, C., Shintake, T., Kitamura, H. \& Kumagai, N. (2012). Nat. Photon. 6, 540-544.

Kameshima, T., Sato, T., Kudo, T., Ono, S., Ozaki, K., Katayama, T., Hatsui, T. \& Yabashi, M. (2016). AIP Conf. Proc. 1741, 040033.

Kim, K. H., Kim, J. G., Nozawa, S., Sato, T., Oang, K. Y., Kim, T. W., Ki, H., Jo, J., Park, S., Song, C., Sato, T., Ogawa, K., Togashi, T., Tono, K., Yabashi, M., Ishikawa, T., Kim, J., Ryoo, R., Kim, J., Ihee, H. \& Adachi, S. (2015). Nature (London), 518, 385-389.

Marinelli, A., Ratner, D., Lutman, A. A., Turner, J., Welch, J., Decker, F.-J., Loos, H., Behrens, C., Gilevich, S., Miahnahri, A. A., Vetter,
S., Maxwell, T. J., Ding, Y., Coffee, R., Wakatsuki, S. \& Huang, Z. (2015). Nat. Commun. 6, 6369.

Osaka, T., Hirano, T., Sano, Y., Inubushi, Y., Matsuyama, S., Tono, K., Ishikawa, T., Yamauchi, K. \& Yabashi, M. (2016). Opt. Express, 24, 9187-9201.

Osaka, T., Yabashi, M., Sano, Y., Tono, K., Inubushi, Y., Sato, T., Matsuyama, S., Ishikawa, T. \& Yamauchi, K. (2013). Opt. Express, 21, 2823-2831.

Roling, S., Zacharias, H., Samoylova, L., Sinn, H., Tschentscher, Th., Chubar, O., Buzmakov, A., Schneidmiller, E., Yurkov, M. V., Siewert, F., Braun, S. \& Gawlitza, P. (2014). Phys. Rev. ST Accel. Beams, 17, 110705.

Roseker, W., Franz, H., Schulte-Schrepping, H., Ehnes, A., Leupold, O., Zontone, F., Lee, S., Robert, A. \& Grübel, G. (2011). J. Synchrotron Rad. 18, 481-491.

Roseker, W., Franz, H., Schulte-Schrepping, H., Ehnes, A., Leupold, O., Zontone, F., Robert, A. \& Grübel, G. (2009). Opt. Lett. 34, $1768-1770$.

Sakamoto, J., Ohwada, K., Ishino, M., Mizuki, J., Ando, M. \& Namikawa, K. (2017). J. Synchrotron Rad. 24, 95-102.

Stetsko, Y. P., Shvyd'ko, Y. V. \& Brian Stephenson, G. (2013). Appl. Phys. Lett. 103, 173508.

Tono, K., Kudo, T., Yabashi, M., Tachibana, T., Feng, Y., Fritz, D., Hastings, J. \& Ishikawa, T. (2011). Rev. Sci. Instrum. 82, 023108.

Tono, K., Togashi, T., Inubushi, Y., Sato, T., Katayama, T., Ogawa, K., Ohashi, H., Kimura, H., Takahashi, S., Takeshita, K., Tomizawa, H., Goto, S., Ishikawa, T. \& Yabashi, M. (2013). New J. Phys. 15, 083035.

Trigo, M., Fuchs, M., Chen, J., Jiang, M. P., Cammarata, M., Fahy, S., Fritz, D. M., Gaffney, K., Ghimire, S., Higginbotham, A., Johnson, S. L., Kozina, M. E., Larsson, J., Lemke, H., Lindenberg, A. M., Ndabashimiye, G., Quirin, F., Sokolowski-Tinten, K., Uher, C., Wang, G., Wark, J. S., Zhu, D. \& Reis, D. A. (2013). Nat. Phys. 9, 790-794.

Yumoto, H., Mimura, H., Koyama, T., Matsuyama, S., Tono, K., Togashi, T., Inubushi, Y., Sato, T., Tanaka, T., Kimura, T., Yokoyama, H., Kim, J., Sano, Y., Hachisu, Y., Yabashi, M., Ohashi, H., Ohmori, H., Ishikawa, T. \& Yamauchi, K. (2012). Nat. Photon. 7, 43-47. 